\title{
EXTENSION OF CONTINUOUS FUNCTIONS TO BAIRE-ONE FUNCTIONS
}

\author{
OLENA KARLOVA
}

\begin{abstract}
We introduce the notion of $B_{1}$-retract and investigate the connection between $B_{1}$ - and $H_{1}$-retracts.
\end{abstract}

\section{INTRODUCTION.}

Recall that a function $f: X \rightarrow Y$ between topological spaces $X$ and $Y$ belongs to the first Baire class or is a Baire-one function, if it is a pointwise limit of a sequence of continuous functions. A function $f$ belongs to the first Lebesgue class or is a Lebesgue-one function if $f^{-1}(F)$ is a $G_{\delta}$-set in $X$ for every closed set $F$ in $Y$. We shall denote by $B_{1}(X, Y)\left(H_{1}(X, Y)\right)$ the collection of all functions of the first Baire (Lebesgue) class from $X$ to $Y$.

K. Kuratowski [9, p. 445] proved that every continuous function $f: E \rightarrow \mathbb{R}$ on an arbitrary subset $E$ of a metric space $X$ can be extended to a Lebesgue-one function on the whole space. According to Lebesgue-Hausdorff Theorem [9, p. 402] the extension is also a Baire-one function.

O. Kalenda and J. Spurný [7] showed that if $E$ is a Lindelöf hereditarily Baire subspace or $E$ is a Lindelöf $G_{\delta}$-subspace of a completely regular space $X$ then every Baire-one function $f: E \rightarrow \mathbb{R}$ can be extended to a Baire-one function on the whole space.

It was proved in [13] that any Baire-one function with values in a $\sigma$-metrizable space with some additional conditions and defined on a Lindelöf $G_{\delta}$-subspace of a normal space can be extended to a Baire-one function on the whole space.

The question about the extension of the class of range spaces of extendable functions naturally arises. In [8] the notion of $H_{1}$-retract was introduced. A subset $E$ of a topological space $X$ is called an $H_{1}$-retract if there exists a Lebesgue-one function $r: X \rightarrow E$ such that $r(x)=x$ for all $x \in E$. It was shown in 8 that $E$ is an $H_{1}$-retract of $X$ iff for any topological space $Y$ and for any continuous function $f: E \rightarrow Y$ there exists an extension $g \in H_{1}(X, Y)$ of $f$. The following result was established in [8].

Theorem 1.1. 8, Corollary 3.3] A set $E$ is an $H_{1}$-retract of a completely metrizable space $X$ if and only if $E$ is a $G_{\delta}$-set in $X$.

In this paper we introduce the notion of $B_{1}$-retract and prove several of its properties. Further, using Theorem 1.1 and the generalization of Lebesgue-Hausdorff Theorem, we find out that $B_{1}$-retracts and $H_{1}$-retracts are tightly connected in many cases. And in the last section we give two examples which show that even for subsets of the plane $\mathbb{R}^{2}$ the notions of retract, $H_{1}$-retract and $B_{1}$-retract are different. 


\section{2. $B_{1}$-RETRACTS AND THEIR PROPERTIES.}

Recall [1] that a subset $E$ of a topological space $X$ is said to be a retract of $X$ if there exists a continuous function $r: X \rightarrow E$ such that $r(x)=x$ for all $x \in E$. The function $r$ is called a retraction of $X$ onto $E$. It is well-known that a set $E \subseteq X$ is a retract of $X$ if and only if for any topological space $Y$ every continuous function $f: E \rightarrow Y$ can be extended to a continuous function $g: X \rightarrow Y$.

We call a subset $E$ of a topological space $X$ a $B_{1}$-retract of $X$ if there exists a Baire-one function $r: X \rightarrow E$ such that $r(x)=x$ for all $x \in E$. We call the function $r$ a $B_{1}$-retraction of $X$ onto $E$.

Note that a composition of a continuous function and a Baire-one function is a Baire-one function. This fact and the definition of a $B_{1}$-retract immediately imply the following proposition.

Proposition 2.1. Let $X$ be a topological space. $A$ set $E \subseteq X$ is a $B_{1}$-retract of $X$ if and only if for any topological space $Y$ every continuous function $f: E \rightarrow Y$ can be extended to a Baire-one function $g: X \rightarrow Y$.

A subset $A$ of a topological space $X$ is called a regular $G_{\delta}-$ set [12] if there exists a sequence $\left(G_{n}\right)_{n=1}^{\infty}$ of open sets in $X$ such that

$$
A=\bigcap_{n=1}^{\infty} G_{n}=\bigcap_{n=1}^{\infty} \bar{G}_{n} .
$$

We say that a topological space $X$ has a regular $G_{\delta}$-diagonal if its diagonal $\Delta=\{(x, x): x \in X\}$ is a regular $G_{\delta}$-set in $X \times X$.

Obviously, every regular $G_{\delta}$-diagonal is closed $G_{\delta}$-set in $X \times X$. Note that every space with a $G_{\delta}$-diagonal is Hausdorff.

Proposition 2.2. Let $X$ be a topological space with a regular $G_{\delta}$-diagonal and $E$ be a $B_{1}$-retract of $X$. Then $E$ is a $G_{\delta}$-set in $X$.

Proof. Since the diagonal $\Delta$ is regular $G_{\delta}$-set, it can be represented as $\Delta=$ $\bigcap_{n=1}^{\infty} G_{n}=\bigcap_{n=1}^{\infty} \bar{G}_{n}$, where $\left(G_{n}\right)_{n=1}^{\infty}$ is a decreasing sequence of open sets in $X \times X$. Let $r: X \stackrel{n=1}{\rightarrow} E$ be a $B_{1}$-retraction of $X$ onto $E$. Consider a function $h: X \rightarrow X \times X$, $h(x)=(r(x), x)$. Then $h \in B_{1}(X, X \times X)$ and $E=h^{-1}(\Delta)$. Let $\left(h_{n}\right)_{n=1}^{\infty}$ be a sequence of continuous functions such that $h_{n}(x) \rightarrow h(x)$ for every $x \in X$. We claim that

$$
h^{-1}(\Delta)=\bigcap_{m=1}^{\infty} \bigcup_{n=m}^{\infty} h_{n}^{-1}\left(G_{m}\right) .
$$

Indeed, let $x \in h^{-1}(\Delta)$ and $m \in \mathbb{N}$. Then $h(x) \in G_{m}$. Since $h_{n}(x) \rightarrow h(x)$, there exists a number $n \geq m$ such that $h_{n}(x) \in G_{m}$. Now let $x$ belongs to the right side of the equality. Then there exists a sequence $\left(n_{m}\right)_{m=1}^{\infty}$ such that $n_{m} \geq m$ and $h_{n_{m}}(x) \in G_{m}$ for every $m \in \mathbb{N}$. Assume that $h(x) \notin \Delta$. Then there exists a number $m_{0}$ such that $h(x) \notin \bar{G}_{m_{0}}$. Since $h_{n}(x) \rightarrow h(x)$, there exists a number $n_{0} \geq m_{0}$ such that $h_{n}(x) \notin \bar{G}_{m_{0}}$ for all $n \geq n_{0}$. In particular, $h_{n_{n_{0}}}(x) \notin G_{m_{0}}$ since $n_{n_{0}} \geq n_{0}$. Taking into account that $G_{n_{0}} \subseteq G_{m_{0}}$, we conclude $h_{n_{n_{0}}}(x) \notin G_{n_{0}}$, a contradiction. Hence, $h(x) \in \Delta$.

Since $G_{m}$ is an open set in $X \times X$ for every $m$ and $h_{n}$ is continuous for every $n$, the set $E=h^{-1}(\Delta)$ is $G_{\delta}$ in $X$. 
Notice that a $B_{1}$-retract, in general, is not a $G_{\delta}$-set. For example, let $X$ be a space of all functions $x:[0,1] \rightarrow[0,1]$ equipped with a topology of pointwise convergence, $x$ be an arbitrary point from $X$ and $E=\{x\}$. Since $X$ is compact and non-metrizable, the diagonal of $X$ is not a $G_{\delta}$-set [4 p. 264], consequently, it is not a regular $G_{\delta}$-set, and $E$ is not a $G_{\delta}$-set in $X$. But, clearly, $E$ is a retract (and, therefore, $E$ is a $B_{1}$-retract) of the space $X$.

In connection with the previous remark the following open question naturally arises.

Question 2.3. Do there exist a Hausdorff space $X$ with a $G_{\delta}$-diagonal, but without a regular $G_{\delta}$-diagonal, and a $B_{1}$-retract of $X$, which is not a $G_{\delta}$-set?

It is well-known that every retract of a connected topological space is also a connected space. The following result states that the same is true for $B_{1}$-retracts.

Proposition 2.4. Let $X$ be a connected topological space and $E$ be a $B_{1}$-retract of $X$. Then $E$ is a connected space.

Proof. Let $r: X \rightarrow E$ be a $B_{1}$-retraction of $X$ onto $E$ and $\left(r_{n}\right)_{n=1}^{\infty}$ be a sequence of continuous functions $r_{n}: X \rightarrow E$ such that $r_{n}(x) \rightarrow r(x)$ for every $x \in X$.

Assume the contrary. Then $E=E_{1} \sqcup E_{2}$, where $E_{1}$ and $E_{2}$ are open in $E$ nonempty sets. Since $r_{n}$ is a continuous function and $X$ is a connected space, the set $B_{n}=r_{n}(X)$ is connected for every $n$. Then $B_{n} \subseteq E_{1}$ or $B_{n} \subseteq E_{2}$ for every $n \in \mathbb{N}$. Choose any $x \in E_{1}$. Then $r_{n}(x) \rightarrow r(x)=x$. Since $E_{1}$ is an open set in $E$, there exists a number $n_{1}$ such that $r_{n}(x) \in E_{1}$ for all $n \geq n_{1}$. Then $r_{n}(x) \in B_{n} \cap E_{1}$, that is, $B_{n} \subseteq E_{1}$ for all $n \geq n_{1}$. Analogously, it can be shown that there exists a number $n_{2} \in \mathbb{N}$ such that $B_{n} \subseteq E_{2}$ for all $n \geq n_{2}$. Hence, $B_{n} \subseteq E_{1} \cap E_{2}$ for all $n \geq \max \left\{n_{1}, n_{2}\right\}$, a contradiction.

A topological space $Y$ is called an equiconnected space 3 if there exists a continuous function $\gamma: Y \times Y \times[0,1] \rightarrow Y$, which for every $y^{\prime}, y^{\prime \prime} \in Y$ and $t \in[0,1]$ satisfies the following properties:

(i) $\gamma\left(y^{\prime}, y^{\prime \prime}, 0\right)=y^{\prime}$

(ii) $\gamma\left(y^{\prime}, y^{\prime \prime}, 1\right)=y^{\prime \prime}$,

(iii) $\gamma\left(y^{\prime}, y^{\prime}, t\right)=y^{\prime}$.

We need the following auxiliary fact from 13 .

Lemma 2.5. 13, Lemma 2.1] Let $X$ be a normal space, $Y$ be an equiconnected space, $\left(F_{i}\right)_{i=1}^{n}$ be disjoint closed sets in $X$ and $g_{i}: X \rightarrow Y$ be a continuous function for every $1 \leq i \leq n$. Then there exists a continuous function $g: X \rightarrow Y$ such that $g(x)=g_{i}(x)$ on $F_{i}$ for every $1 \leq i \leq n$.

Proof. The proof is by induction on $n$. Let $n=2$. Since $F_{1}$ and $F_{2}$ are disjoint and closed, by Urysohn's Lemma [4, p. 41] there exists a continuous function $\varphi: X \rightarrow$ $[0,1]$ such that $\varphi(x)=0$ on $F_{1}$ and $\varphi(x)=1$ on $F_{2}$. The space $Y$ is equiconnected, therefore there exists a continuous function $\gamma: Y \times Y \times[0,1] \rightarrow Y$, which satisfies (i) - (iii). Let

$$
g(x)=\gamma\left(g_{1}(x), g_{2}(x), \varphi(x)\right)
$$

for every $x \in X$. Clearly, $g: X \rightarrow Y$ is continuous. If $x \in F_{1}$, then $\varphi(x)=0$ and $g(x)=g_{1}(x)$. If $x \in F_{2}$, then $\varphi(x)=1$ and $g(x)=g_{2}(x)$. 
Assume the lemma is true for all $1 \leq k<n$. We will prove it for $k=n$. According to the assumption, there exists a continuous function $\tilde{g}: X \rightarrow Y$ such that $\left.\tilde{g}\right|_{F_{i}}=g_{i}$ for every $1 \leq i<n$. Since $F=\bigcup_{i=1}^{n-1} F_{i}$ and $F_{n}$ are disjoint and closed in $X$, by the assumption there exists such a continuous function $g: X \rightarrow Y$ that $\left.g\right|_{F}=\tilde{g}$ and $\left.g\right|_{F_{n}}=g_{n}$. Then $\left.g\right|_{F_{i}}=g_{i}$ for every $1 \leq i \leq n$.

We call a subset $A$ of a topological space $X$ an ambiguous set if it is simultaneously $F_{\sigma}$ and $G_{\delta}$ in $X$. Recall that a topological space $X$ is called perfectly normal if it is normal and every closed subset of $X$ is a $G_{\delta}$-set.

Theorem 2.6. Let $X$ be a perfectly normal space, $E \subseteq X$ be an equiconnected space, $E=\bigcup_{n=1}^{\infty} E_{n}$, and the following conditions hold:

(1) $E_{n} \cap E_{m}=\varnothing$ if $n \neq m$;

(2) $E_{n}$ is an ambiguous set in $E$ for every $n \in \mathbb{N}$;

(3) $E_{n}$ is a $B_{1}$-retract of $X$ for every $n \in \mathbb{N}$;

(4) $E$ is a $G_{\delta}$-set in $X$.

Then $E$ is a $B_{1}$-retract of $X$.

Proof. From the condition (2) and [9, p. 359] it follows that for every $n \in \mathbb{N}$ there exists an ambiguous set $C_{n}$ in $X$ such that $C_{n} \cap E=E_{n}$. Let $D_{1}=C_{1}$ and $D_{n}=C_{n} \backslash \bigcup_{k<n} C_{k}$ if $n \geq 2$. Then $D_{n}$ is an ambiguous set for every $n$. Moreover, $D_{n}$ are disjoint sets and $D_{n} \cap E=E_{n}$ for every $n \in \mathbb{N}$. Since $X \backslash E$ is an $F_{\sigma}$-set in $X$, there exists a sequence $\left(F_{n}\right)_{n=1}^{\infty}$ of closed subsets of $X$ such that $X \backslash E=\bigcup_{n=1}^{\infty} F_{n}$. Let $X_{1}=F_{1} \cup D_{1}$, and $X_{n}=\left(F_{n} \cup D_{n}\right) \backslash\left(\bigcup_{k<n}\left(F_{k} \cup D_{k}\right)\right)$ if $n \geq 2$. Obviously, $X_{n}$ is an ambiguous set in $X$ for every $n, X_{n} \cap X_{m}=\varnothing$ if $n \neq m$, and $X=\bigcup_{n=1}^{\infty} X_{n}$.

We will show that $X_{n} \cap E=E_{n}$ for every $n \in \mathbb{N}$. Indeed, if $x \in X_{n} \cap E$, then

$$
x \in\left(F_{n} \cup D_{n}\right) \cap E=\left(F_{n} \cap E\right) \cup\left(D_{n} \cap E\right)=D_{n} \cap E=E_{n} .
$$

If $x \in E_{n}$, then $x \in D_{n} \cap E$, therefore $x \in D_{n}$ and $x \notin F_{m}$ for all $m$. Moreover, $x \notin D_{k}$ for all $k<n$, since $D_{n} \cap D_{k}=\varnothing$ if $n \neq k$. Hence, $x \in X_{n} \cap E$.

According to (3), there exists a sequence of $B_{1}$-retractions $r_{n}: X \rightarrow E_{n}$. Let $r(x)=r_{n}(x)$ if $x \in X_{n}$. We will show that $r \in B_{1}(X, E)$.

For every $n \in \mathbb{N}$ there exists a sequence $\left(r_{n, m}\right)_{m=1}^{\infty}$ of continuous functions $r_{n, m}: X \rightarrow E_{n}$ such that $\lim _{m \rightarrow \infty} r_{n, m}(x)=r_{n}(x)$ for every $x \in X$. Notice that $\lim _{m \rightarrow \infty} r_{n, m}(x)=r(x)$ on $X_{n}$. The set $X_{n}$ is $F_{\sigma}$, therefore, for every $n$ there exists an increasing sequence $\left(B_{n, m}\right)_{m=1}^{\infty}$ of closed subsets $B_{n, m}$ of $X$ such that $X_{n}=\bigcup_{m=1}^{\infty} B_{n, m}$. Let $A_{n, m}=\varnothing$ if $n>m$, and $A_{n, m}=B_{n, m}$ if $n \leq m$. Then Lemma 2.5 implies that for every $m \in \mathbb{N}$ there exists a continuous function $g_{m}: X \rightarrow E$ such that $\left.g_{m}\right|_{A_{n, m}}=r_{n, m}$ since a family $\left\{A_{n, m}: n \in \mathbb{N}\right\}$ is finite for every $m \in \mathbb{N}$.

It remains to prove that $g_{m}(x) \rightarrow r(x)$ on $X$. Fix $x \in X$. Then $x \in X_{n}$ for some $n \in \mathbb{N}$. The sequence $\left(A_{n, m}\right)_{m=1}^{\infty}$ is increasing, and, in consequence, there exists $m_{0}$ such that $x \in A_{n, m}$ for every $m \geq m_{0}$. Then $g_{m}(x)=r_{n, m}(x)$ for all $m \geq m_{0}$. Hence, $\lim _{m \rightarrow \infty} g_{m}(x)=\lim _{m \rightarrow \infty} r_{n, m}(x)=r(x)$. Therefore, $r \in B_{1}(X, Y)$. 
It is easy to see that $r(x)=x$ for all $x \in E$. Hence, $r$ is a $B_{1}$-retraction of $X$ onto $E$.

\section{The connection between $B_{1}$-Retracts and $H_{1}$-Retracts.}

Recall that a family $\mathcal{A}$ of subsets of a topological space $X$ is discrete if every point $x \in X$ has a neighbourhood $U$ that intersects at most one of the sets $A \in \mathcal{A}$. A family $\mathcal{A}=\left(A_{i}: i \in I\right)$ of subsets of a topological space $X$ is said to be strongly discrete [11] if there is a discrete family $\mathcal{G}=\left(G_{i}: i \in I\right)$ of open sets in $X$ such that $\bar{A}_{i} \subseteq G_{i}$ for any $i \in I$. A family $\mathcal{A}$ is $\sigma$-discrete (strongly $\sigma$-discrete) if it can be represented as the union of countably many discrete (strongly discrete) families in $X$.

A family $\mathcal{B}$ of subsets of topological space $X$ is a base for a function $f: X \rightarrow Y$ if for any open set $V$ in $Y$ there exists a subfamily $\mathcal{B}_{V} \subseteq \mathcal{B}$ such that $f^{-1}(V)=\bigcup \mathcal{B}_{V}$. If $\mathcal{B}$ is (strongly) $\sigma$-discrete then it is called (strongly) $\sigma$-discrete base for $f$ and function $f: X \rightarrow Y$ with (strongly) $\sigma$-discrete base is called (strongly) $\sigma$-discrete function. We shall denote by $\Sigma(X, Y)\left(\Sigma^{*}(X, Y)\right)$ the set of all (strongly) $\sigma$-discrete functions from $X$ to $Y$.

A topological space $X$ is collectionwise normal if $X$ is $T_{1}$-space and for each discrete family $\left(F_{i}: i \in I\right)$ of closed sets there exists a discrete family $\left(G_{i}: i \in I\right)$ of open sets such that $F_{i} \subseteq G_{i}$ for every $i \in I$. It is easy to see that a space is collectionwise normal if and only if every discrete family of its subsets is strongly discrete.

Note that any function with values in a second countable topological space is strongly $\sigma$-discrete. R. Hansell [6 proved that every Lebesgue-one function with a complete metric domain space and a metric range space is $\sigma$-discrete. Taking into account that a complete metric space is collectionwise normal, we obtain the strongly $\sigma$-discreteness of such a function.

Recall that a topological space $X$ is arcwise connected if for any two points $x$ and $y$ from $X$ there exists a continuous function $f:[0,1] \rightarrow X$ such that $f(0)=x$ and $f(1)=y$. A space $X$ is called locally arcwise connected if for every $x \in X$ and for any its neighbourhood $U$ there exists a neighbourhood $V$ of $x$ such that for each $y \in V$ there is a continuous function $f:[0,1] \rightarrow U$ such that $f(0)=x$ and $f(1)=y$.

We shall need the following results of L. Veselý [11] and M. Fosgerau [5] concerning the equality between Baire and Lebesgue classes.

Theorem 3.1. [11, Theorem 3.7(i)] Let $X$ be a normal space, $Y$ be an arcwise connected and locally arcwise connected metric space. Then

$$
H_{1}(X, Y) \cap \Sigma^{*}(X, Y)=B_{1}(X, Y) .
$$

Theorem 3.2. [5, Theorem 2] Let $Y$ be a complete metric space. Then the following conditions are equivalent:

(i) $Y$ is connected and locally connected;

(ii) $H_{1}(X, Y) \cap \Sigma(X, Y)=B_{1}(X, Y)$ for any metric space $X$.

Theorem 3.3. Let $X$ be a normal space and $E$ be an arcwise connected and locally arcwise connected metrizable ambiguous subspace of $X$. If one of the following conditions holds

(i) $E$ is separable, or 
(ii) $X$ is collectionwise normal, then $E$ is a $B_{1}$-retract of $X$.

Proof. Fix any point $x^{*} \in E$ and define

$$
r(x)= \begin{cases}x, & \text { if } x \in E, \\ x^{*}, & \text { if } x \in X \backslash E .\end{cases}
$$

We claim that $r$ is an $H_{1}$-retraction of $X$ onto $E$. Indeed, take an arbitrary open set $V$ in $E$. If $x^{*} \notin V$, then $r^{-1}(V)=V$. Since $E$ is metrizable, $V$ is an $F_{\sigma^{-}}$ set in $E$. Moreover, $E$ is $F_{\sigma}$ in $X$, therefore, $V$ is $F_{\sigma}$ in $X$. If $x^{*} \in V$, then $r^{-1}(V)=V \cup(X \backslash E)$. Since $V$ and $X \backslash E$ are $F_{\sigma^{-}}$-sets in $X, r^{-1}(V)$ is also an $F_{\sigma}$-set in $X$.

(i) Since $E$ is a second countable space, a function $r$ is strongly $\sigma$-discrete. According to Theorem 3.1, $r \in B_{1}(X, E)$.

(ii) Show that $r: X \rightarrow E$ is strongly $\sigma$-discrete. Since $E$ is $F_{\sigma}$ in $X$, there exists an increasing sequence $\left(F_{n}\right)_{n=1}^{\infty}$ of closed subsets of $X$ such that $E=\bigcup_{n=1}^{\infty} F_{n}$. Note that every metrizable space has a $\sigma$-discrete base according to Bing's Theorem [4, p. 282], therefore, for every $n$ we can choose a $\sigma$-discrete base $\mathcal{U}_{n}$ of $F_{n}$. Then $\mathcal{U}_{n}=\bigcup_{m=1}^{\infty} \mathcal{U}_{n, m}$, where $\left(\mathcal{U}_{n, m}\right)_{m=1}^{\infty}$ is a sequence of discrete families in $F_{n}, n \in \mathbb{N}$. The set $F_{n}$ is closed in $X$, and, consequently, $\mathcal{U}_{n, m}$ is discrete in $X, n, m \in \mathbb{N}$. Since $X$ is collectionwise normal, the family $\mathcal{U}_{n, m}$ is strongly discrete in $X$. Then the families $\mathcal{U}_{n}$ and $\mathcal{U}=\bigcup_{n=1}^{\infty} \mathcal{U}_{n}$ are strongly $\sigma$-discrete in $X$. Let $\mathcal{B}=\mathcal{U} \cup\{X \backslash E\}$. Then $\mathcal{B}$ is a strongly $\sigma$-discrete family in $X$.

We prove that $\mathcal{B}$ is a base for $r$. Let $U$ be an open set in $E$. Then $U=$ $\bigcup_{n=1}^{\infty}\left(U \cap F_{n}\right)$. Since $U \cap F_{n}$ is an open set in $F_{n}$ for every $n$, there exists a subfamily $\mathcal{U}_{n, U} \subseteq \mathcal{U}_{n}$ such that $U \cap F_{n}=\bigcup \mathcal{U}_{n, U}$. If $x^{*} \notin U$, then $r^{-1}(U)=U=\bigcup_{n=1}^{\infty} \bigcup \mathcal{U}_{n, U}$. If $x^{*} \in U$, then $r^{-1}(U)=U \cup(X \backslash E)=\bigcup_{n=1}^{\infty} \cup \mathcal{U}_{n, U} \cup(X \backslash E)$. Therefore, $\mathcal{B}$ is a strongly $\sigma$-discrete base for $r$. Hence, $r \in \Sigma^{*}(X, E)$.

By Theorem 3.1, $r \in B_{1}(X, E)$.

Theorem 3.4. Let $X$ be a complete metric space and $E \subseteq X$ be an arcwise connected and locally arcwise connected $G_{\delta}$-set. Then $E$ is a $B_{1}$-retract of $X$.

Proof. Theorem 1.1 implies that there exists an $H_{1}$-retraction $r: X \rightarrow E$ of $X$ onto $E$. Since $X$ is complete, Hansell's Theorem [6, Theorem 3] implies that $r$ is strongly $\sigma$-discrete. According to Theorem 3.1 $r \in B_{1}(X, E)$ and, therefore, $r$ is a $B_{1}$-retraction of $X$ onto $E$.

\section{EXAMPLES.}

It is well-known that any retract of a locally connected space is also a locally connected space, and any retract of an arcwise connected space is an arcwise connected space too. We give an example which shows that for $B_{1}$-retracts it is not true.

We first prove the following auxiliary fact. 
Lemma 4.1. Let $A=[a, b] \times[c, d], B_{1}=A \backslash((a, b) \times(c, d])$ and $B_{2}=A \backslash$ $((a, b) \times[c, d))$. Then $B_{i}$ is a retract of $A$ for every $i=1,2$.

Proof. Let $p_{1}=\left(\frac{a+b}{2}, d+1\right)$ and $p_{2}=\left(\frac{a+b}{2}, c-1\right)$. For $i=1,2$ and $(x, y) \in$ $A$ denote by $\ell_{i}(x, y)$ the line which connects $(x, y)$ and $p_{i}$. Consider a function $\varphi_{i}: A \rightarrow B_{i}$ such that $\varphi_{i}(x, y)$ is the point of the intersection of $\ell_{i}(x, y)$ with $B_{i}$, $i=1,2$. It is easy to see that for every $i=1,2$ the function $\varphi_{i}$ is a retraction of $A$ onto $B_{i}$.

Example 4.2. There exists a connected closed $B_{1}$-retract of $[0,1]^{2}$ which is neither arcwise connected nor locally connected.

Proof. Let

$E=(\{0\} \times[0,1]) \cup\left(\bigcup_{n=1}^{\infty}\left(\left\{\frac{1}{n}\right\} \times[0,1]\right) \cup\left(\left[\frac{1}{2 n}, \frac{1}{2 n-1}\right] \times\{1\}\right) \cup\left(\left[\frac{1}{2 n+1}, \frac{1}{2 n}\right] \times\{0\}\right)\right)$.

It is not difficult to check that $E$ is a connected closed subset of $[0,1]^{2}$, which is neither arcwise connected nor locally connected. We show that $E$ is a $B_{1}$-retract of $[0,1]^{2}$.

Let

$$
\begin{gathered}
A_{n}=\left[\frac{1}{n+1}, \frac{1}{n}\right] \times[0,1], \quad B_{n}=\left[0, \frac{1}{n+1}\right] \times[0,1], \quad n \geq 1, \\
E_{n}=\left(\left[\frac{1}{n+1}, \frac{1}{n}\right] \times[0,1]\right) \backslash\left(\left(\frac{1}{n+1}, \frac{1}{n}\right) \times[0,1)\right) \text { if } n \text { is an odd number, } \\
E_{n}=\left(\left[\frac{1}{n+1}, \frac{1}{n}\right] \times[0,1]\right) \backslash\left(\left(\frac{1}{n+1}, \frac{1}{n}\right) \times(0,1]\right) \text { if } n \text { is an even number. }
\end{gathered}
$$

By Lemma 4.1, $E_{n}$ is a retract of $A_{n}$ for every $n$. Denote by $\varphi_{n}$ a retraction of $A_{n}$ onto $E_{n}, n \in \mathbb{N}$. Let $\psi_{n}$ be a continuous function $\psi_{n}: B_{n} \rightarrow\left\{\frac{1}{n+1}\right\} \times[0,1]$, $\psi_{n}(x, y)=\left(\frac{1}{n+1}, y\right)$.

For every $n \geq 1$ and $x, y \in[0,1]$ define

$$
r_{n}(x, y)= \begin{cases}\varphi_{k}(x, y), & (x, y) \in A_{k}, 1 \leq k \leq n, \\ \psi_{n}(x, y), & (x, y) \in B_{n} .\end{cases}
$$

The function $r_{n}:[0,1]^{2} \rightarrow \bigcup_{k \leq n} E_{k}$ is correctly defined and continuous for every $n$, since $\left.\varphi_{k}\right|_{A_{k} \cap A_{k+1}}=\left.\varphi_{k+1}\right|_{A_{k} \cap A_{k+1}}, 1 \leq k<n$, and $\left.\varphi_{n}\right|_{A_{n} \cap B_{n}}=\left.\psi_{n}\right|_{A_{n} \cap B_{n}}$.

We show that $\left(r_{n}\right)_{n=1}^{\infty}$ is a pointwise convergent sequence on $[0,1]^{2}$. Fix an arbitrary $(x, y) \in[0,1]^{2}$. If $x \neq 0$, then there exists $n_{0}$ such that $(x, y) \in A_{n_{0}}$. Then $r_{n}(x, y)=\varphi_{n_{0}}(x, y)$ for all $n \geq n_{0}$, that is $r_{n}(x, y) \underset{n \rightarrow \infty}{\rightarrow} \varphi_{n_{0}}(x, y)$. Note that if $(x, y) \in E$, then $\varphi_{n_{0}}(x, y)=(x, y)$. If $x=0$, then $r_{n}(x, y)=\psi_{n}(x, y)=$ $\left(\frac{1}{n+1}, y\right) \underset{n \rightarrow \infty}{\rightarrow}(0, y)=(x, y)$. Hence, there exists $\lim _{n \rightarrow \infty} r_{n}(x, y)$ for each $(x, y) \in$ $[0,1]^{2}$. We remark that $\lim _{n \rightarrow \infty} r_{n}(x, y)=(x, y)$ on $E$. Moreover, since $E$ is closed, $\lim _{n \rightarrow \infty} r_{n}(x, y) \in E$ for all $(x, y) \in[0,1]^{2}$.

Set $r(x, y)=\lim _{n \rightarrow \infty} r_{n}(x, y)$ for every $(x, y) \in[0,1]^{2}$. Then $r:[0,1]^{2} \rightarrow E$ is a $B_{1}$-retraction of $[0,1]^{2}$ onto $E$.

Note that for the set $E$ from the previous example there exists an $H_{1}$-retraction $r:[0,1]^{2} \rightarrow E$. Though $E$ is a complete metric separable connected space, we cannot apply Theorem 3.2 for $r$ since $E$ is not locally connected. Therefore, it is natural to ask: is every connected $H_{1}$-retract of a complete metric separable 
connected and locally connected space its $B_{1}$-retract? The following example shows that the answer to this question is negative.

Example 4.3. There exists a connected $H_{1}$-retract of a complete metric separable connected and locally connected space which is not its $B_{1}$-retract.

Proof. Let $\mathbb{Q}_{0}=\mathbb{Q} \cap[0,1]=\left\{q_{n}: n \in \mathbb{N}\right\}$. For every $n \in \mathbb{N}$ consider a function $f_{n}:[0,1] \rightarrow[-1,1], f_{n}(x)=\sin \frac{1}{x-q_{n}}$ if $x \neq q_{n}$, and $f_{n}\left(q_{n}\right)=0$. For every $x \in[0,1]$ define

$$
\begin{gathered}
f(x)=\sum_{n=1}^{\infty} \frac{1}{2^{n}} f_{n}(x), \\
X=[0,1] \times[-1,1] \quad \text { and } E=\operatorname{Gr}(f)=\{(x, y) \in X: y=f(x)\} .
\end{gathered}
$$

For every $n$ the function $f_{n}:[0,1] \rightarrow[-1,1]$ belongs to the first Baire class, since it is discontinuous only in one point $x=q_{n}$ (see for instance [10, p. 384]). Moreover, $f_{n}$ is a Darboux function (i.e. $f_{n}(A)$ is connected for every connected set $A \subseteq[0,1])[2$, p. 13]. Then $f$ is a Darboux Baire-one function, as the sum of the uniform convergent series of Darboux Baire-one functions [2, p. 13]. Therefore, the set $E$, as the graph of $f$, is connected [2, p. 9] and $G_{\delta}[9$, p. 393] in $X$. Hence, according to Theorem 1.1 the set $E$ is an $H_{1}$-retract of $X$.

We prove that $E$ is not a $B_{1}$-retract of $X$. Assume that there exists a function $r \in B_{1}(X, E)$ such that $r(p)=p$ for all $p \in E$. Let $\left(r_{n}\right)_{n=1}^{\infty}$ be a sequence of continuous functions $r_{n}: X \rightarrow E$, which is pointwise convergent to $r$ on $X$. Since $X$ is compact and connected, $E_{n}=r_{n}(X)$ is also compact and connected for every $n$. Note that at least one of $E_{n}$ contains more than one point. Indeed, assume that all the sets $E_{n}$ consist of one point, i.e. $E_{n}=\left\{p_{n}\right\}$, where $p_{n} \in E, n \in \mathbb{N}$. Choose two different points $p^{\prime}$ and $p^{\prime \prime}$ from $E$. Then $p_{n}=r_{n}\left(p^{\prime}\right) \underset{n \rightarrow \infty}{\rightarrow} p^{\prime}$ and $p_{n}=r_{n}\left(p^{\prime \prime}\right) \underset{n \rightarrow \infty}{\rightarrow} p^{\prime \prime}$, a contradiction. Hence, there exists a number $n_{0}$ such that $E_{n_{0}}$ contains at least two different points (to be more precise, the cardinality of $E_{n_{0}}$ is equal to $\mathfrak{c}$ since $E_{n_{0}}$ is a connected set).

Now fix $p, q \in E_{n_{0}}$. Since $E_{n_{0}} \subseteq \operatorname{Gr}(f)$, the points $p$ and $q$ are represented as $p=(a, f(a))$ and $q=(b, f(b))$. Without loss of generality we can assume that $a<b$. Note that $(x, f(x)) \in E_{n_{0}}$ for any $x \in(a, b)$. Indeed, if there exists a point $x_{0} \in(a, b)$ such that $\left(x_{0}, f\left(x_{0}\right)\right) \notin E_{n_{0}}$, then the line $x=x_{0}$ does not intersect $E_{n_{0}}$. Then, since $E_{n_{0}}$ is connected, it should be completely contained either in the left hand half-plane, or in the right hand half-plane with respect to the line $x=x_{0}$. But this contradicts the fact that both $p$ and $q$ belong to $E_{n_{0}}$.

Since $\mathbb{Q}_{0}$ is dense in $[0,1]$, there exists a number $k$ such that $q_{k} \in(a, b)$. Note that $f$ is discontinuous in every point of $\mathbb{Q}_{0}$, in particular, it is discontinuous in $q_{k}$. Then there exists a sequence $\left(x_{n}\right)_{n=1}^{\infty}, x_{n} \in(a, b)$, such that $\lim _{n \rightarrow \infty} x_{n}=q_{k}$, but $\lim _{n \rightarrow \infty} f\left(x_{n}\right) \neq f\left(q_{k}\right)$. Since $\left(x_{n}, f\left(x_{n}\right)\right) \in E_{n_{0}}$ for every $n$ and $E_{n_{0}}$ is closed, the point $\left(\lim _{n \rightarrow \infty} x_{n}, \lim _{n \rightarrow \infty} f\left(x_{n}\right)\right)=\left(q_{k}, \lim _{n \rightarrow \infty} f\left(x_{n}\right)\right)$ also belongs to $E_{n_{0}}$. But then it must be equal to $\left(q_{k}, f\left(q_{k}\right)\right)$, a contradiction.

Hence, $E$ is not a $B_{1}$-retract of $X$.

\section{REFERENCES}

[1] K. Borsuk, Theory of Retracts, Mir, Moscow, 1971. (Russian).

[2] A. Bruckner, Differentiation of Real Functions [2nd ed.], Providence, RI: American Mathematical Society, 1994. 
[3] J. Dugundji, Locally equiconnected spaces and absolute neghborhood retracts, Fund. Math., 57 (1965), 187-193.

[4] R. Engelking, General Topology, Revised and completed edition. Heldermann Verlag, Berlin, 1989.

[5] M. Fosgerau, When are Borel functions Baire functions?, Fund. Math., 143 (1993), 137-152.

[6] R. Hansell, Borel measurable mappings for nonseparable metric spaces, Trans. Amer. Math. Soc., 161 (1971), 145-168.

[7] O. Kalenda, J. Spurný, Extending Baire-one functions on topological spaces, Topol. Appl., 149 (2005), 195-216.

[8] O. Karlova, Extension of continuous mappings and $H_{1}$-retracts, Bull. Aust. Math. Soc., 78(3) (2008), 497-506.

[9] K. Kuratowski, Topology, T.1, Mir, Moscow, 1966. (Russian).

[10] I. Natanson, Theory of Real Functions, Nauka, Moscow, 1974. (Russian).

[11] L. Veselý, Characterization of Baire-one functions between topological spaces, Acta Univ. Carol., Math. Phys., 33(2) (1992), 143-156.

[12] P. Zenor, On spaces with regular $G_{\delta}$-diagonals, Pacific. J. Math., 40 (1972), 759-763.

[13] T. Zolotukhina, O. Karlova, O. Sobchuk, On the extension of Baire-one functions with values in $\sigma$-metrizable spaces, Ukr. Math. Bull., 2(5) (2008), 280-287. (Ukrainian). 